\title{
Averaging Schwarzschild spacetime
}

\author{
S. Ph. Tegai* and I. V. Drobov' \\ Siberian Federal University, Svobodny av., 79, Krasnoyarsk, 660041
}

(Dated: June 27, 2017)

\begin{abstract}
We tried to average the Schwarzschild solution for the gravitational point source by analogy with the same problem in Newtonian gravity or electrostatics. We expected to get a similar result, consisting of two parts: a smoothed interior part being a sphere filled with some matter content and an empty exterior part described by the original solution. We considered several variants of generally covariant averaging schemes. The averaging of the connection in the spirit of Zalaletdinov's macroscopic gravity gave unsatisfactory results. With the transport operators proposed in the literature it did not give the expected Schwarzschild solution in the exterior part of the averaged spacetime. We were able to construct a transport operator which preserves the Newtonian analogy for the outward region but such an operator does not have a clear geometrical meaning. In contrast, using the curvature as the primary averaged object instead of the connection does give the desired result for the exterior part of the problem in a fine way. However for the interior part, this curvature averaging does not work because the Schwarzschild curvature components diverge as $1 / r^{3}$ near the center and therefore are not integrable.

PACS numbers: 04.20.Jb, 98.80.Jk
\end{abstract}

*Electronic address: tegai_s_f@inbox.ru

${ }^{\dagger}$ Electronic address: drivvl@mail.ru 


\section{INTRODUCTION}

Averaging the Newton potential over spheres centered at different points yields the solution of the Poisson equation for the sphere of uniform density. The exterior part of the solution is again the Newton potential. Now we want to do the same exercise but with the point source solution in general relativity. However this is not so obvious because the generally covariant averaging procedure itself is not yet fully established. Different kinds of averaging schemes have been proposed since the pioneering work of Shirokov and Fisher [1] (see the review by Clifton [2] and references therein). All of them are divided into two groups: spacetime averaging and spatial averaging. The latter is widely used in cosmology, which is the main source of interest in averaging. The most well studied approach was proposed by Buchert [3] and a more recent one was suggested by Sussmann [4]. However those methods aim at the study of the model expansion governed by the analogue of the Friedmann equations, and therefore are not very suitable for our little problem.

The first fully covariant and exact spacetime averaging procedure was developed by Zalaletdinov [5]. His method is based on two principles. One is the so called rule of averaging. To find the average of a tensor field $T_{\beta_{1} \ldots \beta_{m}}^{\alpha_{1} \ldots \alpha_{n}}$ at some point $x$ lying in the domain of averaging $\Sigma$ the field is transported from all the points of $\Sigma$ to $x$ with the help of a bilocal averaging operator $A_{\alpha^{\prime}}^{\alpha}\left(x, x^{\prime}\right)$. Then the result is integrated over $\Sigma$ and divided by its volume

$$
\bar{T}_{\beta_{1} \ldots \beta_{m}}^{\alpha_{1} \ldots \alpha_{n}}=\frac{1}{V_{\Sigma}} \int_{\Sigma} A_{\alpha_{1}^{\prime}}^{\alpha_{1}} \ldots A_{\alpha_{n}^{\prime}}^{\alpha_{n}} A_{\beta_{1}}^{\beta_{1}^{\prime}} \ldots A_{\beta_{m}}^{\beta_{m}^{\prime}} T_{\beta_{1}^{\prime} \ldots \beta_{m}^{\prime}}^{\alpha_{1}^{\prime} \ldots \alpha_{n}^{\prime}} d \Omega^{\prime} .
$$

The second principle is that the average of the connection forms of the original spacetime are declared to be the connection forms of the averaged spacetime. In other words, the primary averaged object of the method is the connection.

Relying on these assumptions, Zalaletdinov derived the system of Macroscopic Gravity equations by applying his averaging procedure to the Einstein equations. Though the resulting equations are rather complicated, the solutions for several important cases were found. First is the solution for a point source [6, 7] and the others are spatially homogeneous solutions [7-10]. The latter is significant for cosmology because the governing equations for the model evolution differ from the Friedmann equations by the additional backreaction term which depends on the scale factor in the same manner as the spatial curvature [8, 11]. This means that the dynamical curvature parameter is decoupled from the geometrical value of 
the curvature, distorting the luminosity distance-redshift relation. The quantitative observational consequences were studied by Clarkson, Clifton, Coley, and Sung [12] and later by Wijenayake, Lin, and Ishak [13].

Zalaletdinov's Macroscopic Gravity is not the only possible spacetime method of averaging. Several other covariant methods have been proposed. They differ in their rules and objects of averaging. Behrend [14] suggests transporting tensor quantities by means of the relativistic analogue of the Wegner-Wilson line operator. The primary averaged objects are tetrads. Another idea is to average a set of scalar invariants sufficient to completely determine the spacetime. These can be the curvature invariants constructed from the Riemann tensor and its derivatives [15] or the Cartan scalars [16]. The advantage of such a method is the simplicity of the averaging rule for the scalars, which do not require any transportation. Brannlund, Coley, and van den Hoogen [17] consider the possibility of averaging the curvature forms instead of the connection forms. They use the same averaging rule as in the theory of Macroscopic Gravity, but with the specific transport operator corresponding to the Weitzenböck connection.

A very different scheme was proposed by Green and Wald [18]. It has recently been widely discussed [19 22] because of the restrictive conclusion on possible backreaction. The method generalizes the approach of Burnett [23] and Isaacson [24] for gravitationally radiating systems and defines the average metric as the limit of a one-parameter family of metrics without the usual integration over a finite volume.

With such a variety of different methods it is important to probe them with some relatively simple tests, that is, to take an exact microscopic solution and then average it properly. For example, applying any averaging procedure to the already smooth solutions such as the constant curvature spacetimes should not change the source. In this paper we suggest testing some of the proposed methods by averaging the Schwarzschild solution for the point gravitational source and comparing the results with a similar Newtonian problem.

\section{SPHERICALLY SYMMETRIC ANSATZ IN CARTESIAN COORDINATES}

In Cartesian coordinates, a basis of 1-forms for the static spherically symmetric spacetime can always be written as

$$
\theta^{(0)}=\vartheta_{1}(r) d t
$$




$$
\theta^{(i)}=\vartheta_{2}(r) \frac{x^{i}}{r} d t+\left[\vartheta_{3}(r) \frac{x^{i} x_{j}}{r^{2}}+\vartheta_{4}(r)\left(\delta_{j}^{i}-\frac{x^{i} x_{j}}{r^{2}}\right)\right] d x^{j}
$$

where $r=\sqrt{x^{2}+y^{2}+z^{2}}$. The Latin indices span the $x, y, z$ coordinates and are lowered and raised by the Kronecker symbol.

For Schwarzschild spacetime, the coefficients take the form

$$
\vartheta_{1}=\vartheta_{3}=\vartheta_{4}=1, \quad \vartheta_{2}=\sqrt{2 m / r}
$$

which leads to the linear element

$$
d s^{2}=\left(1-\frac{2 m}{r}\right) d t^{2}-2 \sqrt{\frac{2 m}{r}} \frac{x_{i}}{r} d t d x^{i}-\delta_{i j} d x^{i} d x^{j}
$$

The metric determinant $g$ is constant $(g=-1)$, so the chosen coordinates are volume preserving.

The connection forms for the general static spherically symmetric spacetime have the following structure

$$
\begin{gathered}
\omega_{(i)}^{(0)}=\gamma_{1}(r) \frac{x_{i}}{r} d t+\gamma_{2}(r) \frac{x_{i} x_{j}}{r^{2}} d x^{j}+\gamma_{3}(r) \delta_{i j} d x^{j} \\
\omega_{(j)}^{(i)}=\gamma_{4}(r)\left(\delta_{k}^{i} \frac{x_{j}}{r}-\delta_{j k} \frac{x^{i}}{r}\right) d x^{k} .
\end{gathered}
$$

For the Schwarzschild solution

$$
\gamma_{1}=\frac{m}{r^{2}}, \quad \gamma_{2}=-\frac{3}{2} \gamma_{3}=\frac{3 \sqrt{2 m}}{2 r^{3 / 2}}, \quad \gamma_{4}=0
$$

The coefficients of the basis 1-forms $\theta^{(\alpha)}$ and the connection forms $\omega_{(\beta)}^{(\alpha)}$ are related by the first Cartan structure equations

$$
\begin{gathered}
\vartheta_{1}^{\prime}+\left(\gamma_{2}+\gamma_{3}\right) \vartheta_{2}-\gamma_{1} \vartheta_{3}=0, \\
r \gamma_{3} \vartheta_{1}+\vartheta_{2}=0 \\
\vartheta_{2}^{\prime}+\gamma_{2} \vartheta_{1}-\frac{1}{r} \vartheta_{2}=0 \\
\vartheta_{4}^{\prime}-\frac{1}{r}\left(\vartheta_{3}-\vartheta_{4}\right)=0 .
\end{gathered}
$$

The idea of Zalaletdinov's macroscopic gravity [5] is that the averages of the connection forms are declared to be the connection forms of the averaged spacetime. The averaging over a region $\Sigma$ is performed by the rule (1) applied to the connection

$$
\bar{\omega}_{(\beta) \gamma^{\prime}}^{(\alpha)}\left(x^{\prime}\right)=\frac{1}{V_{\Sigma}} \int_{\Sigma} \omega_{(\beta) \gamma}^{(\alpha)}(x) A_{\gamma^{\prime}}^{\gamma}\left(x, x^{\prime}\right) d \Omega,
$$


where $V_{\Sigma}$ is the volume of the averaging region $\Sigma$ and $d \Omega$ is the volume element.

The averaging over an arbitrary volume is very complicated and not related to the Newtonian analogy. So we choose a particular kind of averaging regions, in the form of the 4-cylinders

$$
\Sigma\left(t^{\prime}, \mathbf{r}^{\prime}\right)=\left\{(t, \mathbf{r}) \| t-t^{\prime}|<T / 2,| \mathbf{r}-\mathbf{r}^{\prime} \mid<R\right\}
$$

If we choose the averaging operator $A_{\alpha^{\prime}}^{\alpha}\left(x, x^{\prime}\right)$ to be independent of $t$, then the averaging becomes effectively 3-dimensional, and the averaging regions will be spheres of the same radius $R$ centered at the variable points $\mathbf{r}^{\prime}$.

\section{CONNECTION AVERAGING WITH SOME PARTICULAR CHOICES OF THE AVERAGING OPERATOR}

The most natural choice for the averaging operator is to make it equal to the coordination bivector, which in the volume preserving coordinates is equal to the Kronecker symbol

$$
A_{\beta}^{\alpha}=\delta_{\beta}^{\alpha}
$$

In that case, the average connection forms will be

$$
\begin{gathered}
\bar{\gamma}_{1}=\left\{\begin{array}{c}
m r / R^{3}, \quad r<R \\
m / r^{2}, \quad r>R,
\end{array}\right. \\
\bar{\gamma}_{3}=\frac{2 \sqrt{2 m} \frac{15 r^{3} R^{3}}{}\left[(2 R-r)(R-2 r)(R+r)^{5 / 2}\right.}{\left.-(2 R+r)(R+2 r)|R-r|^{5 / 2}\right],} \\
\bar{\gamma}_{2}=r \bar{\gamma}_{3}^{\prime} .
\end{gathered}
$$

Knowing the connection, we can find the properties of the averaged spacetime. First of all, we see that it fulfills the same ansatz as the original spacetime. Therefore we can say that all the symmetries are preserved. The Killing vector $\xi=\partial / \partial t$ has the norm

$$
\xi^{\alpha} \xi_{\alpha}=1-r^{2} \bar{\gamma}_{3}^{2}
$$

For a sufficiently large radius of the averaging region $(R \gtrsim 0.85 \mathrm{~m})$, the norm becomes positive everywhere and the spacetime will be globally static. For radii less than critical, the norm has two roots. 
The averaged spacetime is divided into exterior and interior parts by the surface $r=R$. The matching conditions require the continuity of the coefficient $\bar{\vartheta}_{4}$ through the matching surface. This gives the boundary conditions for solving Eq. 12 in the interior region. The integration constant in the exterior solution is found from the limit $R \rightarrow 0$, which should yield the Schwarzshild value $\vartheta_{4}=1$. With this, all the tetrads (2), (3) for the averaged spacetime can be found explicitly and even expressed in elementary functions, though the result is cumbersome.

The resulting Einstein tensor has two pairs of eigenvalues

$$
\begin{gathered}
\lambda_{1}=\lambda_{2}=-\frac{2 \bar{\gamma}_{1}}{r \bar{\vartheta}_{4}}+\frac{\bar{\gamma}_{3}^{2}}{\bar{\vartheta}_{4}^{2}}, \\
\lambda_{3}=\lambda_{4}=\frac{\bar{\gamma}_{1} \bar{\gamma}_{1}^{\prime}}{r \bar{\gamma}_{3}\left(\bar{\gamma}_{2}+\bar{\gamma}_{3}\right)}-\frac{2 \bar{\gamma}_{1}}{r \bar{\vartheta}_{4}} .
\end{gathered}
$$

Unfortunately, the denominator $\bar{\gamma}_{2}+\bar{\gamma}_{3}$ turns to zero. It follows directly from Eqs. (17), (18) that the condition $\bar{\gamma}_{2}+\bar{\gamma}_{3}=0$ is equivalent to

$$
75 \frac{r^{6}}{R^{6}}-135 \frac{r^{4}}{R^{4}}+64=0
$$

which has two positive roots in the exterior region $r>R$ leading to the discontinuities in the Einstein tensor. Therefore the result of the averaging is unsatisfactory.

At large distances from a black hole, the series for the eigenvalues take the form

$$
\begin{aligned}
& \lambda_{1}=\lambda_{2} \approx \frac{m}{r^{3}}\left(-\frac{18}{5} \frac{R^{2}}{r^{2}}+O\left(\frac{R^{4}}{r^{4}}\right)\right), \\
& \lambda_{3}=\lambda_{4} \approx \frac{m}{r^{3}}\left(\frac{27}{5} \frac{R^{2}}{r^{2}}+O\left(\frac{R^{4}}{r^{4}}\right)\right) .
\end{aligned}
$$

This behavior is in agreement with the "no backreaction" theorem of Green and Wald [18]. Indeed, the curvature length scale for Schwarzschild spacetime is $m / r^{3}$. Far away from the center, the theorem's conditions are clearly fulfilled and both $\left|\lambda_{1}\right|$ and $\left|\lambda_{3}\right|$ are much smaller than $m / r^{3}$. However we can not really say that there is no backreaction as the initial stress-energy tensor was equal to zero and no correction can be called small compared to it.

In their recent paper, Brannlund, van den Hoogen, and Coley [17] suggested the averaging operator

$$
A_{\alpha^{\prime}}^{\alpha}\left(x, x^{\prime}\right)=e_{(\beta)}^{\alpha}(x) e_{\alpha^{\prime}}^{(\beta)}\left(x^{\prime}\right)
$$

corresponding to the parallel transport of the averaged tensors with the curvature-free Weitzenböck connection. 
The averaging of the connection forms with the help of this operator yields the same results (17), (18) for $\bar{\gamma}_{3}(r)$ and $\bar{\gamma}_{2}(r)$. The $\bar{\gamma}_{1}(r)$ coefficient is different and can be written as

$$
\bar{\gamma}_{1}=\sqrt{\frac{2 m}{r}}\left(\bar{\gamma}_{2}+\bar{\gamma}_{3}\right) .
$$

The expressions for the Einstein tensor's eigenvalues (20) and 21) also hold true but due to the $\bar{\gamma}_{2}+\bar{\gamma}_{3}$ factor in $\bar{\gamma}_{1}$, all the eigenvalues are finite for any $r>R$. The behavior of the eigenvalues near the center is given by the series

$$
\begin{gathered}
\lambda_{1}=\frac{128 m}{9 \pi R^{3}}\left[\sqrt{\frac{R}{r}}-\frac{27}{40}\left(\frac{r}{R}\right)^{3 / 2}+O\left(\left(\frac{r}{R}\right)^{2}\right)\right], \\
\lambda_{3}=-\frac{m}{R^{3}}\left[\frac{R^{3}}{r^{3}}-\frac{9}{4} \cdot \frac{R}{r}+O(1)\right] .
\end{gathered}
$$

While at infinity, the leading terms of the expansion are

$$
\begin{aligned}
& \lambda_{1}=\lambda_{2} \approx \frac{m}{r^{3}}\left(-\frac{18}{5} \frac{R^{2}}{r^{2}}+O\left(\frac{R^{4}}{r^{4}}\right)\right), \\
& \lambda_{3}=\lambda_{4} \approx \frac{m}{r^{3}}\left(\frac{18}{5} \frac{R^{2}}{r^{2}}+O\left(\frac{R^{4}}{r^{4}}\right)\right) .
\end{aligned}
$$

We see that this choice of the averaging operator satisfies the Green and Wald theorem in the same way. It is not fully satisfactory either, because the singularity at the center remains after the averaging.

For both considered choices of the operator $A_{\beta}^{\alpha}$, the exterior region of the averaged spacetime is no longer described by the Schwarzschild solution. This contrasts with the Newtonian case, where the exterior potential remains the same after the averaging.

\section{EMPTY EXTERIOR AVERAGED SPACETIME}

We now try to construct an averaging operator that will give us the Schwarzschild solution for the exterior part of the averaged spacetime. Mars and Zalaletdinov [25] showed that the general averaging operator can be factorized as

$$
A_{\beta}^{\alpha}\left(x^{\prime}, x\right)=F_{\gamma}^{\alpha}(x) F_{\beta}^{-1 \gamma}\left(x^{\prime}\right) .
$$

For the averaged spacetime to coincide with the original, it is sufficient to require the integrand in the averaging rule 13 to be harmonic, i.e.,

$$
\Delta\left(\omega_{(\beta) \sigma}^{(\alpha)}(x) F_{\gamma}^{\sigma}(x)\right)=0 .
$$


We were able to find a particular solution of this equation in the form

$$
F_{0}^{0}=1, \quad F_{i}^{0}=f_{1}(r) \frac{x_{i}}{r}, \quad F_{0}^{i}=0, \quad F_{j}^{i}=f_{2}(r) \delta_{j}^{i}
$$

where

$$
\begin{gathered}
f_{1}=C_{1} r^{-1}+C_{2} r+C_{3} r^{2}+C_{4} r^{4}, \\
f_{2}=\frac{1}{3} \cdot \sqrt{\frac{2 m}{r}}\left(C_{1} r^{-1}-C_{2} r-C_{3} r^{2}+C_{4} r^{4}\right) .
\end{gathered}
$$

Therefore, the averaging operator (31) with the components

$$
\begin{gathered}
A_{0}^{0}=1, \quad A_{i}^{0}=\frac{f_{1}(r)}{f_{2}\left(r^{\prime}\right)} \cdot \frac{x_{i}}{r}-\frac{f_{1}\left(r^{\prime}\right)}{f_{2}\left(r^{\prime}\right)} \cdot \frac{x_{i}^{\prime}}{r^{\prime}}, \\
A_{0}^{i}=0, \quad A_{j}^{i}=\frac{f_{2}(r)}{f_{2}\left(r^{\prime}\right)} \delta_{j}^{i}
\end{gathered}
$$

with $f_{1}, f_{2}$ as in Eqs. (34) and (35) does give the Schwarzschild solution in the exterior region of the averaged spacetime.

\section{CURVATURE AVERAGING}

Another idea suggested by Brannlund, Coley, and van den Hoogen [17] is that the proper averaging object might not be the connection forms, but rather the curvature forms. This proposition, together with their choice of the averaging operator in the form (25), works especially well for the spacetimes of constant curvature. The action of the operator (25) on the curvature forms yields

$$
\begin{aligned}
A_{\mu}^{\mu^{\prime}}\left(x, x^{\prime}\right) A_{\nu}^{\nu^{\prime}}\left(x, x^{\prime}\right) \mathcal{R}_{.(\beta) \mu^{\prime} \nu^{\prime}}^{(\alpha)}\left(x^{\prime}\right) & =e_{(\sigma)}^{\mu^{\prime}}\left(x^{\prime}\right) e_{\mu}^{(\sigma)}(x) e_{(\rho)}^{\nu^{\prime}}\left(x^{\prime}\right) e_{\nu}^{(\rho)}(x) \mathcal{R}_{.(\beta) \mu^{\prime} \nu^{\prime}}^{(\alpha)}\left(x^{\prime}\right) \\
& =e_{\mu}^{(\sigma)}(x) e_{\nu}^{(\rho)}(x) \mathcal{R}_{.(\beta)(\sigma)(\rho)}^{(\alpha)}\left(x^{\prime}\right)
\end{aligned}
$$

For constant curvature spacetimes, the Riemann tensor can be expressed through the metric as

$$
R_{\mu \nu \alpha \beta}=R\left(g_{\mu \alpha} g_{\nu \beta}-g_{\mu \beta} g_{\nu \alpha}\right) .
$$

Its tetrad components are constant and therefore the averaging does not change the initial curvature forms. In contrast, averaging the connection forms does change a constant curvature spacetime, as was shown in the same paper [17] on a two-dimensional example. 
The nontrivial tetrad components of the Riemann tensor for Schwarzschild spacetime are given by

$$
\begin{gathered}
R_{.(i)(0)(j)}^{(0)}=\frac{m}{r^{3}}\left(3 \frac{x_{i} x_{j}}{r^{2}}-\delta_{i j}\right), \\
R_{.(j)(k)(l)}^{(i)}=\frac{m}{r^{3}}\left[2 \delta_{j[l} \delta_{k]}^{i}+3\left(\frac{x^{i} x_{[l}}{r^{2}} \delta_{j k]}-\frac{x_{j} x_{[l}}{r^{2}} \delta_{k]}^{i}\right)\right] .
\end{gathered}
$$

It is straightforward to check that all of them are harmonic functions at any point besides the center. Thus the average of the curvature form over any domain that does not contain the center point will give the original curvature form by the mean value property of harmonic functions.

For the domains which do contain the center, the Riemann tensor's components 40, 41) are not integrable, due to the $1 / r^{3}$ factor.

\section{CONCLUDING REMARKS}

We have considered the averaging of Schwarzschild spacetime in the sense of Zalaletdinov's macroscopic gravity with several types of averaging operator. None of the addressed variants are free from certain flaws. The operators of the form $A_{\alpha^{\prime}}^{\alpha}\left(x, x^{\prime}\right)=\delta_{\alpha^{\prime}}^{\alpha}$ and $A_{\alpha^{\prime}}^{\alpha}\left(x, x^{\prime}\right)=$ $e_{(\beta)}^{\alpha}(x) e_{\alpha^{\prime}}^{(\beta)}\left(x^{\prime}\right)$ lead to singularities in the averaged spacetimes. In addition, the exterior regions of the spacetimes averaged with the help of those operators are not empty, with a negative effective energy density. The operator (31) with the components (36) and (37) does provide the same behavior of averaging as in the electrostatics analogy, but its geometrical interpretation is unclear. Therefore it is difficult to apply it to spacetimes other than that of Schwarzschild.

At the same time, curvature averaging gives the clear expected result for the exterior part of the considered problem. This supports the conjecture that the appropriate object for the averaging should be the curvature but not the connection. The violation of the integrability for the interior part of the problem can probably be explained by the poor choice of the integration volume (14). Indeed, the coordinate $t$ does not have the meaning of a time variable over the entirety of a domain containing the center point. Under the horizon, the integration goes over the timelike hypersurface, breaking the desired Newtonian analogy.

At large distances from the center, our results agree with the Green and Wald "no backreaction" theorem in the sense that the arising effective stress-energy tensor is small compared 
to the curvature length scale. And there is indeed no backreaction when we apply the ways of averaging that maintain the Newtonian analogy and produce the same Schwarzschild spacetime as the result. But in Zalaletdinov's averaging scheme with the first two considered transport operators, one can not say that there is no backreaction. The reason is that the initial spacetime is empty and so no correction to its stress-energy tensor can be negligible. The direct application of the method of Green and Wald is difficult in our problem, even in regions remote from the center, because the result heavily depends on the choice of parametrization, as was shown in the criticism by Buchert et al. [21]. For example, let us introduce the Green and Wald parameter $\lambda$ in such a way that the Schwarzschild mass $m$ is equal to the sum $m_{1}+\lambda$. Then the metric (5) can be decomposed as $g_{\alpha \beta}=g_{\alpha \beta}^{(0)}+h_{\alpha \beta}$ where $h_{\alpha \beta}$ is small when we consider only large distances from the center and $g_{\alpha \beta}^{(0)}$, which is called the averaged metric, is the Schwarzschild solution with an arbitrary mass $m_{1}$. So the result of such an averaging is much too arbitrary, ranging from Minkowski spacetime $\left(m_{1}=0\right)$ to the unchanged initial spacetime $\left(m_{1}=m\right)$, though the difference in the stress-energy tensor for different choices of $m_{1}$ is zero, in perfect agreement with the results of Green and Wald.

[1] M. F. Shirokov and I. Z. Fisher Astron. Zh. 39, 899 (1962).

[2] T. Clifton Int. J. Mod. Phys. D 22, 1330004 (2013).

[3] T. Buchert Gen. Rel. Grav. 32, 105 (2000); 33, 1381 (2001).

[4] R. Sussman Class. Quantum Grav. 30, 065015 (2013).

[5] R. M. Zalaletdinov Gen. Rel. Grav. 24, 1015 (1992); 25, 673 (1993).

[6] R. J. van den Hoogen Gen. Rel. Grav. 40, 2213 (2008).

[7] R. J. van den Hoogen AIP Conf. Proc. 1122, 408 (2009).

[8] A. A. Coley, N. Pelavas, and R. M. Zalaletdinov Phys Rev. Lett. 95, 151102 (2005).

[9] R. J. van den Hoogen J. Math. Phys. 50, 082503 (2009).

[10] T. Wijenayake and M. Ishak Phys. Rev. D 91, 063534 (2015).

[11] A. A. Coley and N. Pelavas Phys. Rev. D 74087301 (2006); 75, 043506 (2007).

[12] C. Clarkson, T. Clifton, A. A. Coley, and R. Sung Phys. Rev. D 85, 043506 (2012).

[13] T. Wijenayake, W. Lin, and M. Ishak Phys. Rev. D 94, 083501 (2016).

[14] J. Behrend arXiv:0812.2859v2 [gr-qc] (2008). 
[15] A. A. Coley Class. Quantum Grav. 27, 245017 (2010).

[16] P. Kašpar and O. Svítek Class. Quantum Grav. 31, 095012 (2014).

[17] J. Brannlund, R. van den Hoogen, and A. Coley Int. J. Mod. Phys. D 19, 1915 (2010).

[18] S. R. Green and R. M. Wald, Phys. Rev. D 83, 084020 (2011); 87, 124037 (2013); Class. Quantum Grav. 33, 125027 (2016).

[19] G. F. R. Ellis Class. Quantum Grav. 28, 164001 (2011).

[20] S. J. Szybka, K. Głód, M. J. Wyrębowski, and A. Konieczny Phys. Rev. D 89, 044033 (2014).

[21] T. Buchert et al., Class. Quantum Grav. 32, 215021 (2015).

[22] K. Bolejko and M. Korzyński, Int. J. Mod. Phys. D, 26, 1730011 (2017).

[23] G. A. Burnett J. Math. Phys. (N.Y.) 30, 90 (1989).

[24] R. A. Isaacson Phys. Rev. 166, 1263 (1968); 1661272 (1968).

[25] M. Mars and R. M. Zalaletdinov J. Math. Phys. 38, 4741 (1997). 